\title{
Padrões de comportamento ingestivo de cordeiras recebendo ou não suplemento em pastagem de milheto
}

\author{
Ingestive behavior patterns of ewe lambs receiving or not supplement on Pearl millet pasture
}

\author{
Anna Carolina Cerato Confortin' Carolina Bremm ${ }^{\text {II }}$ Marta Gomes da Rocha ${ }^{\text {III }}$ \\ José Henrique Souza da Silva ${ }^{I}$ Denise Adelaide Gomes Elejalde ${ }^{I I}$ Daniele Gindri Camargo \\ Aline Tatiane Nunes da Rosa ${ }^{I}$
}

RESUMO

O comportamento ingestivo e os padrões de deslocamento e procura de forragem de cordeiras em pastagem de milheto (Pennisetum glaucum (L.)) foram avaliados quando estas foram mantidas exclusivamente em pastagem ou em pastagem recebendo suplemento (ração comercial fornecida diariamente às $17 \mathrm{~h} . \mathrm{O}$ delineamento experimental foi inteiramente casualizado, com duas repetições de área. Os animais que receberam suplemento reduziram o tempo de pastejo e aumentaram o tempo destinado às outras atividades. Os padrões de ingestão, deslocamento e procura de forragem foram alterados pelas mudanças na estrutura do pasto ao longo dos estádios de desenvolvimento do milheto e não foram modificados pelo recebimento de suplemento.

Palavras-chave: estratégias alimentares, lotação contínua, Pennisetum glaucum.

\section{ABSTRACT}

The ingestive behavior and displacement and forage searching patterns of female lambs in Pearl millet (Pennisetum glaucum (L.)) were evaluated when lambs were kept exclusively on Pearl millet pasture and on pearl millet pasture receiving supplement. The supplement used was a commercial ration given daily at $17 \mathrm{~h}$. The experimental design was completely randomized, with two area replicates. The animals that received supplement reduced the grazing time and increased the idling time. The intake, displacement and forage searching patterns were influenced by changing on pasture structure along the Pearl millet stage of development and were not modified by supplement supply.

Key words: continuous stocking, feed strategies, Pennisetum glaucum.

\section{INTRODUÇÃO}

As forrageiras cultivadas de estação quente são espécies que conciliam alta produção de matéria seca com qualidade de forragem. Dentre elas, o milheto (Pennisetum glaucum (L.)) é a forrageira anual de verão mais utilizada no Rio Grande do Sul (PEDROSO et al., 2009). Sua utilização, em sistemas de produção de ovinos, juntamente com o fornecimento de suplementos, é uma alternativa para acelerar o desenvolvimento dos animais nascidos no período de inverno.

A resposta dos animais em pastejo ao recebimento de suplementos pode variar com as características da pastagem, por meio de mudanças na relação planta-animal-suplemento. Variações nas características vegetativas do pasto podem ter grande efeito no comportamento de animais em pastejo, e devem ser levadas em consideração quando programas de suplementação são avaliados (KRYSL \& HESS, 1993).

Uma mesma massa de forragem pode determinar diferentes taxas de ingestão de forragem (DEMMENT \& LACA, 1993), promovendo alterações no comportamento ingestivo dos animais em pastejo. A altura, a densidade, as diferentes partes da planta, a composição botânica do dossel e o arranjo espacial são fatores que afetam a ingestão e a digestão de

IPrograma de Pós-graduação em Zootecnia, Universidade Federal de Santa Maria (UFSM), Santa Maria, RS, Brasil.

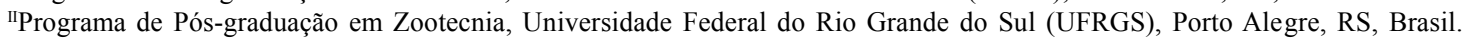

IIIDepartamento de Zootecnia, Universidade Federal de Santa Maria (UFSM), 97105-900, Santa Maria, RS, Brasil. E-mail: mgdarocha@gmail.com. Autor para correspondência. 
plantas forrageiras, interferindo diretamente no comportamento ingestivo de herbívoros (SOLLENBERGER\&BURNS, 2001).

As decisões dos animais são organizadas de forma hierárquica e tomadas em diferentes escalas, iniciando no âmbito da paisagem, passando pelos níveis de comunidade, patch, estação alimentar e planta, até chegar ao bocado (CARVALHO, 1997). A decisão de colheita de um bocado, dentre vários outros possíveis, é decorrente da movimentação do animal ao longo do ambiente de pastejo e de todos os processos sensoriais e cognitivos envolvidos (UNGAR, 1996). Essas respostas dos animais frente ao ambiente de pastejo podem ser definidas por padrões de distribuição e associação no espaço, que são detectados por meio de análises multivariadas de classificação e ordenação (PILLAR, 1998).

Objetivou-se avaliar os padrões de distribuição espacial do comportamento ingestivo de cordeiras mantidas exclusivamente em pastagem de milheto ou recebendo suplemento.

\section{MATERIAL E MÉTODOS}

O experimento foi realizado no período de 01/02 a 07/04/2006, em área do Departamento de Zootecnia da Universidade Federal de Santa Maria, situada na Depressão Central do Rio Grande do Sul. O delineamento experimental foi inteiramente casualizado, com medidas repetidas no tempo, com quatro tratamentos e duas repetições de área, totalizando oito unidades experimentais, com aproximadamente $0,07 \mathrm{ha}$ cada.

O milheto foi semeado de forma convencional com densidade de semeadura de $30 \mathrm{~kg}$ $\mathrm{ha}^{-1}$ de sementes viáveis. A adubação consistiu de $250 \mathrm{~kg} \mathrm{ha}^{-1}$ de N-P-K da fórmula 05-20-20 e, em cobertura, foram adicionados $100 \mathrm{~kg} \mathrm{ha}^{-1} \mathrm{de} \mathrm{N}$, na forma de uréia. A área experimental utilizada apresentava um banco de sementes de papuã (Urochloa plantaginea), o que ocasionou a presença dessa espécie na composição da pastagem.

Avaliou-se diferentes estratégias alimentares: $\mathrm{SS}$ - animais exclusivamente em pastagem de milheto (Pennisetum glaucum (L.)); S0,5 - animais recebendo suplemento na proporção de $0,5 \%$ do peso corporal (PC); S1,0 - animais recebendo suplemento na proporção de 1,0\% do PC; S1,5 - animais recebendo suplemento na proporção de $1,5 \%$ do PC. O suplemento utilizado (ração comercial contendo 88\% de MS; $21,6 \%$ de PB; $17,9 \%$ de FDN) foi composto por $34 \%$ de farelo de soja, $29 \%$ de farelo de trigo, $30 \%$ de farelo de arroz integral, melaço, casca de arroz moída, calcário calcítico, fosfato bicálcico, cloreto de sódio e premix vitamínico mineral, fornecido diariamente, às 17:00h, em baias individualizadas, localizadas dentro dos piquetes. Após 30 minutos, os animais eram retirados e as sobras eram recolhidas e pesadas. Todos os animais tiveram livre acesso à água e sal mineral.

Os animais utilizados foram cordeiras, produtos do cruzamento entre as raças Ile de France e Texel, com idade e peso médio inicial de 6,5 meses e $28,7 \mathrm{~kg}$, respectivamente. O método de pastejo foi de lotação contínua, com número variável de animais para manter uma massa de forragem de, em média, de $2000 \mathrm{~kg}$ $\mathrm{ha}^{-1}$ de MS. O ajuste de lotação foi feito semanalmente, utilizando o método proposto por HERINGER \& CARVALHO (2002). Os animais foram submetidos a um período de adaptação de dez dias aos níveis de suplemento e a um período de pastejo de 64 dias, o qual foi dividido em três períodos: 1 - de $02 / 02$ a 22/02; 2 - de 23/02 a 15/03 e 3 - de 16/03 a 06/04. O pastejo foi diurno, entre $8 \mathrm{~h}$ e $17 \mathrm{~h}$ e $30 \mathrm{~min}$., com permanência dos animais em abrigos no período noturno.

A cada dez dias, a massa de forragem, em kg $\mathrm{ha}^{-1}$ de MS, foi avaliada, por meio da técnica de estimativa visual com dupla amostragem (MANNETJE, 2000), com cinco cortes rentes ao solo e 20 estimativas visuais. A forragem proveniente dos cortes foi homogeneizada e dividida em duas subamostras: uma utilizada para a determinação do teor de MS do pasto e outra para separação botânica e morfológica (lâminas foliares, colmos + bainhas e material senescente). Todas as amostras foram pesadas e secas em estufa, com circulação de ar forçada a $55^{\circ} \mathrm{C}$, por no mínimo 72 horas. Por meio do produto da massa de forragem e da participação percentual dos componentes morfológicos do pasto, obteve-se a massa de forragem verde (lâminas foliares e colmos + bainhas; MFV), a massa de lâminas foliares e a massa de colmos + bainhas e, por meio da divisão entre essas duas ultimas variáveis, obteve-se a relação lâmina foliar: colmo + bainha (RFC).

Nas mesmas datas em que foi estimada a massa de forragem, foi mensurada a altura do dossel, considerada a medida da distância do solo até a altura média de curvatura das folhas do pasto. A taxa de acúmulo diário de forragem $\left(\mathrm{kg} \mathrm{ha}^{-1} \mathrm{de} \mathrm{MS}\right)$ foi estimada com o uso de gaiolas de exclusão ao pastejo, avaliadas a cada 21 dias. A oferta de forragem $(\mathrm{kg}$ de MS $100 \mathrm{~kg}$ de $\left.\mathrm{PV}^{-1}\right)$ foi calculada da seguinte forma: ((massa de forragem do período/número de dias + taxa de acúmulo diário de forragem/taxa de lotação)*100) e esse valor multiplicado pelo percentual de lâminas foliares na MF permitiu obter a oferta de lâminas foliares verdes (OFL). 
As observações do comportamento ingestivo foram realizadas no período diurno, das $8 \mathrm{~h}$ e $30 \mathrm{~min}$. às $17 \mathrm{~h}$ e $30 \mathrm{~min}$., sendo três avaliações $(17 / 02$, $09 / 03$ e 05/04/06). Foram utilizados avaliadores treinados para a observação dos animais-teste de cada unidade experimental. As anotações foram feitas a cada dez minutos, por meio de observação visual (JAMIESON \& HODGSON, 1979a), sendo registradas as atividades de pastejo, ruminação, permanência no cocho e outras atividades. Durante os mesmos períodos de avaliação do comportamento ingestivo, quando os animais estavam em atividade de pastejo, foram registradas, a cada dez minutos, as taxas de bocado dos animaisteste, considerada como o tempo gasto pelo animal para realizar 20 bocados (HODGSON, 1982).

Nos períodos do dia de maior frequencia de pastejo, foi anotado o tempo que o animal levava para visitar dez estações alimentares e o número de passos realizados entre elas. Esses valores foram posteriormente transformados para estações alimentares visitadas por minuto e número de passos por minuto. Uma estação alimentar foi definida como o espaço correspondente ao pastejo sem movimentos das patas dianteiras (LACA et al., 1992), enquanto que um passo foi definido como cada movimento das patas dianteiras.

Para a determinação do consumo, foi utilizado, como indicador externo, $1 \mathrm{~g}$ de óxido de cromo $\left(\mathrm{Cr}_{3} \mathrm{O}_{2}\right)$, fornecido aos animais-teste por via oral. Foram realizadas três determinações no decorrer do ciclo do milheto, sendo uma em cada período de pastejo. O consumo de matéria seca foi estimado pela fórmula: consumo de matéria seca=produção fecal/1digestibilidade in vitro da matéria orgânica e a produção fecal, em $\mathrm{kg}$ de MS $\mathrm{dia}^{-1}$, foi estimada por meio da fórmula: produção fecal $=$ cromo administrado $\left(\mathrm{g} \mathrm{dia}^{-1}\right) /$ cromo nas fezes ( $\mathrm{g} \mathrm{kg}^{-1}$ de MS) (POND et al., 1989). Para o cálculo da massa do bocado, dividiu-se o consumo diário de forragem, expresso em kg animal ${ }^{-1}$, pelo total de bocados diários obtidos por taxa de bocado $\times$ tempo de pastejo (JAMIESON \& HODGSON, 1979b).

Os dados de comportamento ingestivo e padrões de deslocamento e procura pela forragem foram submetidos a análises de variância e regressão polinomial, por meio do pacote estatístico SAS, versão 8.2 (2001) e à análise de variância com testes de aleatorização com 10000 interações, ambos a 5\% de significância. Foram realizados testes de contrastes entre os tratamentos pastagem de milheto ("Pasto") e pastagem de milheto com suplementação ("Suplemento"). Para detectar variações no comportamento ingestivo das cordeiras e na composição botânica e morfológica do pasto ao longo dos períodos de pastejo, utilizou-se análise multivariada de ordenação pelo método de coordenadas principais; a medida de semelhança adotada foi a distância euclidiana e utilizou-se o pacote estatístico MULTIV (PILLAR, 2004).

\section{RESULTADOS E DISCUSSÃO}

As variáveis de comportamento ingestivo e padrões de deslocamento e procura não se ajustaram a nenhum modelo de regressão $(\mathrm{P}>0,05)$ em função dos níveis de suplemento. Por isso, os dados foram discutidos com base no contraste entre cordeiras mantidas exclusivamente em pastagem de milheto ("Pasto") e cordeiras em pastagem de milheto recebendo suplemento ("Suplemento").

Não houve interação estratégias alimentares $\times$ períodos de avaliação para as variáveis de comportamento ingestivo e padrões de deslocamento e procura. As cordeiras que não receberam suplemento pastejaram por mais tempo (Tabela 1) que os animais suplementados, despendendo 68,42 minutos a mais com o pastejo. O manejo para o consumo de suplemento explica $43,85 \%$ da redução no tempo de pastejo das cordeiras suplementadas, pois as cordeiras permaneciam presas em baias individualizadas por 30 minutos para consumir o suplemento. Também o maior aporte de nutrientes proveniente do suplemento pode ter ocasionado essa redução, a qual, de acordo com PATIÑO PARDO et al. (2003), passa a ter importância no desempenho animal, à medida que pode implicar redução no gasto de energia associado ao pastejo.

Em azevém anual, BREMM et al. (2008) também observaram redução no tempo de pastejo de cordeiras desmamadas, em decorrência da suplementação. Essa redução foi atribuída ao aumento da eficiência de colheita de nutrientes do pasto pelos animais que receberam suplemento, segundo definição de KRYSL \& HESS (1993). No presente experimento, contudo, essa hipótese não se aplica, uma vez que as cordeiras mantidas exclusivamente em pastagem de milheto realizaram bocados de massa semelhante às que receberam suplemento (Tabela 1).

O tempo de ruminação foi semelhante entre animais exclusivamente em pastejo e recebendo suplemento (Tabela 1). Já o tempo de outras atividades foi reduzido quando houve aumento no tempo de pastejo, evidenciando o caráter excludente do repertório de atividades diárias dos animais (CARVALHO et al., 2001); assim, observou-se que as cordeiras permaneceram mais tempo em outras atividades quando receberam suplemento.

Ciência Rural, v.40, n.12, dez, 2010. 
Tabela 1 - Comportamento ingestivo de cordeiras e padrões de ingestão, deslocamento e procura por forragem, quando mantidas exclusivamente em pastagem (Pasto) ou recebendo suplemento (Suplemento).

\begin{tabular}{|c|c|c|c|}
\hline \multirow{2}{*}{ Variáveis } & & ratégias aliment & \multirow[b]{2}{*}{ Suplemento } \\
\hline & \multicolumn{2}{|l|}{ Pasto } & \\
\hline Tempo de pastejo (min.). & \multicolumn{2}{|l|}{444,37 a } & $375,95 \mathrm{~b}$ \\
\hline Tempo de ruminação (min.). & \multicolumn{2}{|l|}{49,37} & 62,70 \\
\hline Tempo de outras atividades (min.). & \multicolumn{2}{|l|}{$46,24 \mathrm{~b}$} & $70,20 \mathrm{a}$ \\
\hline Taxa de bocados (bocados $\min ^{-1}$ ). & \multicolumn{2}{|l|}{32,72} & 34,61 \\
\hline Massa de bocado (g bocado ${ }^{-1} \mathrm{MS}$ ). & \multicolumn{2}{|l|}{0,06} & 0,08 \\
\hline Passos $\min ^{-1}$ (número). & \multicolumn{2}{|l|}{12,86} & 13,04 \\
\hline Estações $\min ^{-1}$ (número). & \multicolumn{2}{|l|}{8,95} & 8,86 \\
\hline \multirow[t]{2}{*}{ Consumo total de MS dia ${ }^{-1}(\mathrm{~kg})$. } & \multicolumn{2}{|l|}{1,10} & 1,34 \\
\hline & $02 / 02-22 / 02$ & $23 / 02-15 / 03$ & $16 / 03-06 / 04$ \\
\hline Tempo de pastejo (min.). & $403,99 \mathrm{a}$ & $371,24 \mathrm{~b}$ & 409,37 a \\
\hline Tempo de ruminação (min.). & 51,66 & 62,08 & 66,87 \\
\hline Tempo de outras atividades (min.). & $59,57 \mathrm{~b}$ & 84,15 a & $41,24 \mathrm{~b}$ \\
\hline Taxa de bocados (bocados $\min ^{-1}$ ). & $29,61 \mathrm{c}$ & $35,99 \mathrm{~b}$ & 38,15 a \\
\hline Massa de bocado (g bocado ${ }^{-1} \mathrm{MS}$ ). & $0,11 \mathrm{a}$ & $0,05 \mathrm{c}$ & $0,07 \mathrm{~b}$ \\
\hline Passos $\min ^{-1}$ (número). & $14,52 \mathrm{a}$ & $12,45 \mathrm{~b}$ & $11,52 \mathrm{~b}$ \\
\hline Estações $\min ^{-1}$ (número). & $10,50 \mathrm{a}$ & $7,75 \mathrm{~b}$ & $8,15 \mathrm{~b}$ \\
\hline Consumo total de MS dia ${ }^{-1}(\mathrm{~kg})$. & $1,87 \mathrm{a}$ & $0,88 \mathrm{~b}$ & $1,08 \mathrm{~b}$ \\
\hline
\end{tabular}

Médias seguidas de letras distintas na mesma linha diferem entre si pelo teste de aleatorização, com 10000 interações $(\mathrm{P}<0,05)$.

Exclusivamente em pastagem ou recebendo suplemento, as cordeiras apresentaram semelhantes número de estações alimentares minuto ${ }^{-1}$, passos minuto $^{-1}$ e taxa de bocados, que corresponderam a, em média, 8,88 estações, 12,99 passos e 34,14 bocados minuto $^{-1}$, respectivamente (Tabela 1). A estrutura do dossel foi modificada ao longo do período de utilização da pastagem (Figura 1): a altura do dossel foi maior no início da utilização da pastagem (Período 1: de 02/02 a $22 / 02)$ e esses valores de altura do dossel $(25,8 \mathrm{~cm})$, provavelmente são em decorrência da maior presença do milheto, cuja proporção na massa de forragem verde passou de $82,5 \%$ no início da utilização da pastagem para $15,0 \%$ no final do período. Ao longo do período de pastejo houve aumento na proporção de papuã, que variou de $16,3 \%$ (no início) a 25,6\% (no final) na massa de forragem verde, além de um aumento na proporção de outras espécies, principalmente poaia (Richardia brasilienses), que variou de 0,0 a 63,4\%.

A estrutura do pasto nos períodos de avaliação exerceu influência sobre o comportamento ingestivo e padrões de deslocamento e procura por alimento das cordeiras submetidas a diferentes estratégias alimentares (Tabela 1). Isso confirma que os padrões de regulação do consumo dos animais em pastejo normalmente são afetados pela participação dos componentes morfológicos e botânicos presentes no pasto, que podem se apresentar nas mais diferentes formas ao animal (HOBBS et al., 2003). No primeiro período, a estrutura do pasto, com elevada proporção de milheto e maior altura do dossel, permitiu que as cordeiras colhessem bocados de maior massa. Isso ocorre porque a profundidade do bocado, principal componente da massa de bocado, é diretamente correlacionada com a altura do pasto e tem sido relatada como proporção constante (entre 35 e $50 \%$ ) do comprimento do perfilho estendido (CARVALHO, 2007). Assim, uma vez que a taxa de ingestão instantânea de forragem tende a aumentar progressivamente com a elevação da massa de bocado (HODGSON et al., 1997), os valores superiores de massa de bocados, nesse período, resultaram em maior consumo de forragem (Tabela 1).

No início do pastejo, as cordeiras também realizaram maior número de passos $\mathrm{min}^{-1} \mathrm{e}$ visitaram mais estações $\min ^{-1}$ (Tabela 1). Como a massa de bocado guarda estreita relação com a disponibilidade e qualidade de forragem, provavelmente o maior número 


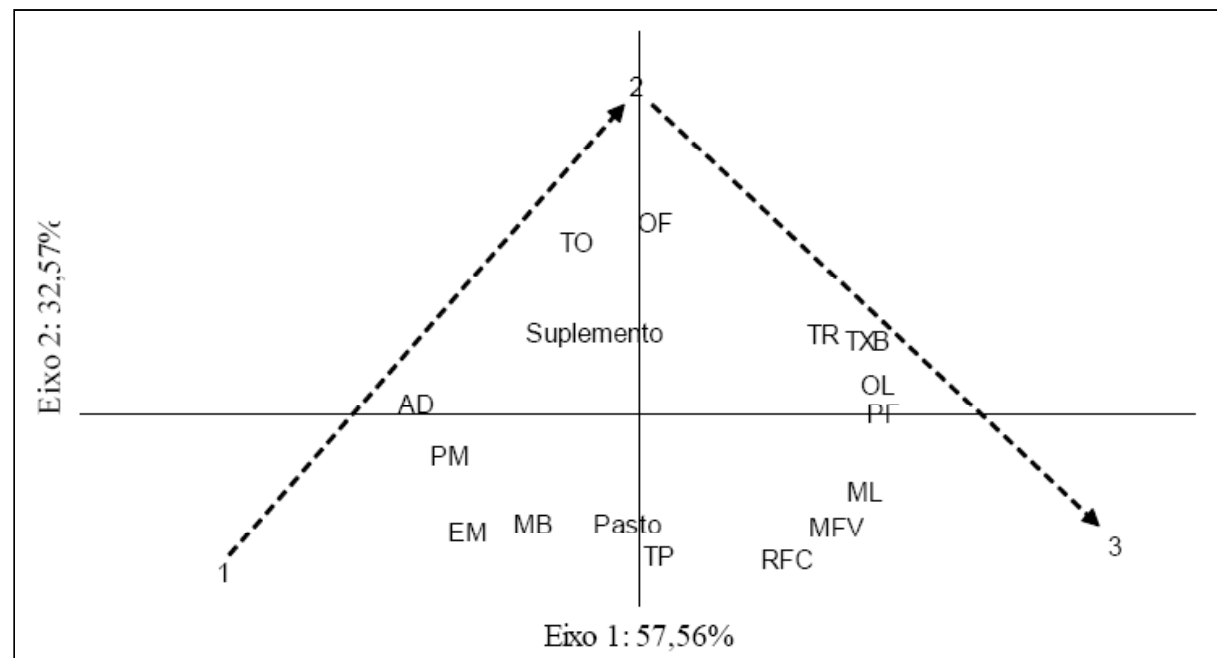

Figura 1 - Diagrama de ordenação de variáveis estruturais do pasto: altura do dossel (AD), massa de forragem verde (MFV), massa de lâminas foliares (ML), oferta de forragem (OF), oferta de lâminas foliares $(\mathrm{OL})$, perdas de forragem $(\mathrm{PF})$ e relação lâmina foliar/colmo+bainha (RFC); e do comportamento ingestivo: estações alimentares por minuto (EM), massa de bocados (MB), taxa de bocados (TXB), tempo de pastejo (TP), tempo de ruminação (TR), tempo de outras atividades (TO) e passos por minutos (PM) de cordeiras mantidas exclusivamente em pastagem de milheto (Pasto) ou recebendo suplemento (Suplemento), de acordo com os períodos de pastejo (1, 2 e 3). Os eixos I e II representam $90,13 \%$ da variação total dos dados.

de estações alimentares visitadas foi favorecido pela percepção de melhores oportunidades de consumo em outros locais (CHARNOV, 1976). Também o maior número de passos $\min ^{-1}$ demonstra uma melhor condição de seleção de forragem, pois, de acordo com PRACHE \& ROGUET (1996), em condições de menor disponibilidade de forragem, os animais caminham menos entre estações alimentares sucessivas.

A taxa de bocados foi inferior no primeiro período de avaliação, o que ocorreu em resposta à maior massa de bocados coletados pelas cordeiras nesse período. Quando o herbívoro apreende mais forragem por bocado, mais tempo é destinado para manipular essa massa ingerida. Essa relação negativa entre a taxa e a massa de bocado, bem como a relação negativa entre a taxa de bocados e a altura do dossel podem ser observadas no diagrama de ordenação (Figura 1). O que se vê nesse diagrama pode ser explicado pelo fato de que, em dosséis de menores alturas, os animais coletam bocados de massa reduzida e, visando buscar a manutenção da ingestão de nutrientes, aumentam o número de bocados por minuto. Já à medida que a altura do pasto aumenta, um número menor de bocados é necessário para compor uma mesma dieta, ou maior será o intervalo de tempo para realizar um novo bocado, em virtude da maior massa a ser mastigada e consumida (CASTRO, 2002).
No segundo período de avaliação (de 23/02 a 15/03), embora houvesse maior oferta de forragem, o pasto apresentava uma estrutura desfavorável ao pastejo, pois, além da redução na proporção de milheto na composição da pastagem, o milheto presente estava passando para o estádio de florescimento e a poaia ainda não apresentava grande participação na massa de forragem $(26,4 \%)$. Essa condição resultou em menor massa e taxa de bocados, menor número de estações alimentares e passos realizados a cada minuto. O tempo de pastejo inferior, nesse período, pode ser uma resposta à estrutura desfavorável para a seleção e apreensão de forragem, pois a regulação do tempo que o animal destina ao pastejo é baseada no balanço energético feito pelo próprio animal, o qual pode reduzir seu tempo de pastejo quando considerar anti-econômico prosseguir (ROOK, 2000).

Observa-se no diagrama de ordenação que no terceiro período de avaliação foram obtidos os maiores valores de massa de forragem verde, massa de lâminas foliares, relação lâmina foliar: colmo+bainha (Figura 1). Embora essas características sejam geralmente correlacionadas positivamente com a massa de bocado e o ganho de peso, de acordo com HODGSON et al. (1994), quando são considerados os prováveis efeitos sobre a massa do bocado, a profundidade do estrato de lâminas pode ser uma 
melhor descrição das condições da pastagem do que sua altura ou mesmo a massa de forragem (PALHANO et al., 2002). Assim, a estrutura do pasto no terceiro período, composta principalmente por Poaia $(63,4 \%)$ e com a presença de outras espécies rosuladas, pode explicar os baixos valores de massa de bocado e os maiores valores de taxas de bocado nesse período.

O tempo de ruminação foi semelhante entre períodos de avaliação. Esse é um componente importante do repertório comportamental dos ruminantes porque determina o potencial máximo do tempo de pastejo, que é dado pela relação entre a taxa de ingestão de forragem e a taxa de digestão de forragem (SEARLE et al., 2007). Como o tempo de digestão aumenta quando a qualidade da dieta diminui (DEMMENT \& VAN SOEST, 1985), um tempo de ruminação semelhante pode significar que as cordeiras colheram forragem com qualidade também semelhante, independentemente do período de utilização da pastagem.

\section{CONCLUSÃO}

O fornecimento de suplemento para cordeiras reduz o tempo diurno de pastejo e aumenta o tempo dedicado a outras atividades, sem alterar seus padrões de ingestão, deslocamento e procura. Os padrões de comportamento ingestivo de animais sob estratégias alimentares recebendo ou não suplementos são dependentes das características estruturais do pasto ao longo do período de pastejo ao qual são submetidos.

\section{REFERÊNCIAS}

BREMM, C. et al. Comportamento ingestivo de ovelhas e cordeiras em pastagem de azevém anual sob níveis crescentes de suplementação. Revista Brasileira de Zootecnia, v.37, n.12, p.2097-2106, 2008. Disponível em: <http://www.scielo.br/ s c i e lo.ph p s c r i p t = s c i_pdf\& p id = S 1516 $35982008001200004 \& \operatorname{lng}=$ en $\& n r m=i s o \& t \operatorname{lng}=\mathrm{pt}>$. Acesso em: 12 set. 2010 . doi: $10.1590 / \mathrm{S} 1516-35982008001200004$.

CARVALHO, P.C.F. A estrutura da pastagem e o comportamento ingestivo de ruminantes em pastejo. In: SIMPÓSIO SOBRE AVALIAÇÃO DE PASTAGENS COM ANIMAIS, 2., 1997, Maringá. Anais... Maringá: Universidade Estadual de Maringá, 1997. p.25-52.

CARVALHO, P.C.F. et al. Importância da estrutura da pastagem na ingestão e seleção de dietas pelo animal em pastejo. In: REUNIÃO ANUAL DA SOCIEDADE BRASILEIRA DE ZOOTECNIA, 38., 2001, Piracicaba. Anais... Piracicaba: Sociedade Brasileira de Zootecnia, 2001. p.853-871.

CARVALHO, P.C.F. Consumo de forragens por bovinos em pastejo. In: PEDREIRA, C.G.S. et al. (Org.). Produção de ruminantes em pastagens. Piracicaba: FEALQ, 2007. p.177-218.
CASTRO, C.R.C. Relações planta-animal em pastagem de milheto (Pennisetum clandestinum (L.) Leeke) manejadas em diferentes alturas com ovinos. 2002. $200 \mathrm{f}$. Dissertação (Mestrado em Zootecnia) - Universidade Federal do Rio Grande do Sul, Porto Alegre, RS.

CHARNOV, E.L. Optimal foraging: the marginal value theorem. Theoretical Population Biology, v.9, p.129-136, 1976.

DEMMENT, M.W.; LACA, E.A. The grazing ruminant: models and experimental techniques to relate sward structure and intake. In: WORLD CONFERENCE ON ANIMAL PRODUCTION, 7., 1993, Edmonton. Proceedings... Edmonton: Keeling \& Mundi, 1993. p.439-460.

DEMMENT, M.W.; VAN SOEST, P.J. A nutritional explanation for body-size patterns of ruminant and nonruminant herbivores. American Naturalist, v.125, p.641-672, 1985.

HERINGER, I.; CARVALHO, P.C.F. Ajuste da carga animal em experimentos de pastejo: uma nova proposta. Ciência Rural, v.32, n.4, p.675-679, 2002. Disponível em: <http://www.scielo.br/ s cielo.php? s cript=s ci arttext\&pid=S 0103 $84782002000400021 \& \operatorname{lng}=$ en\&nrm $=$ iso $>$. Acesso em: 12 set. 2010. doi: $10.1590 / \mathrm{S} 0103-84782002000400021$.

HOBBS, N.T. et al. Herbivore functional response in heterogeneous environments: a contest among models. Ecology, Ecological Society of America, v.84, p.666-681, 2003.

HODGSON, J. Ingestive behavior. In: LEAVER, J.D. (Ed.). Herbage intake handbook. Hurley: British Grassland Society, 1982. p.113.

HODGSON, J.c et al. Foraging behavior in grazing animals and its impact on plant communities. In: FAHEY, G.C. National conference on forage quality, evaluation and utilization. Nebraska: University of Nebrasca, 1994. p.796-827.

HODGSON, J. et al. Research on foraging behaviour: progress and priorities. In: INTERNATIONAL GRASSLANDS CONGRESS, 18., 1997, Calagary. Proceedings... Calagary: Association Management Centre, 1997. CD-ROM.

JAMIESON, W.S.; HODGSON, J. The effect of daily herbage allowance and sward characteristics upon the ingestive behavior of calves under strip-grazing management. Grass and Forage Science, v.34, p.261-271, 1979a.

JAMIESON, W.S.; HODGSON, J. The effect of variation in sward characteristics upon the ingestive behavior and herbage intake of calves and lambs under continuous stocking management. Grass and Forage Science, v.34, p.273-281, 1979b.

KRYSL, L.J.; HESS, B.W. Influence of supplementation on behavior of grazing cattle. Journal of Animal Science, v.71, p.2546-2555, 1993.

LACA, E.A. al. An integrated methodology for studying shortterm grazing behaviour of cattle. Grass and Forage Science, v.47, p.81-90, 1992.

MANNETJE, L.t' Measuring biomass of grassland vegetation. In: MANNETJE, L.t'; JONES, R.M. (Eds.). Field and 
laboratory methods for grassland and animal production research. Cambridge: CABI, 2000. p.151-178.

PALHANO, A.L. et al. Influência da estrutura da pastagem na geometria do bocado e nos processos de procura e manipulação da forragem. Ciência e Cultura, n.31, FACIAG 02, p.33-52, 2002. Disponível em: <http://www.utp.br/tuiuticienciaecultura/FCA/ FCA\%2031/PDF/art3.pdf>. Acesso em: 12 set. 2010.

PATIÑO PARDO, N.M. et al. Comportamento ingestivo diurno de novilhos em pastejo submetidos a níveis crescentes de suplementação energética. Revista Brasileira de Zootecnia, v.32, n.6, p.1408-1418, 2003. Disponível em: <http:// www.scielo.br/scielo.php?script $=$ sci_arttext\&pid $=\mathrm{S} 1516-$ $35982003000600016 \& \operatorname{lng}=\mathrm{en} \& \mathrm{nrm}=\mathrm{iso}>$. Acesso em: $12 \mathrm{set}$. 2010. 1590/S1516-35982003000600016.

PEDROSO, C. E. S. et al. Características morfogênicas de milheto sob lotação rotacionada com diferentes períodos de descanso. Revista Brasileira de Zootecnia, v.38, n.12, p.2311-2319, 2009. Disponível em: <http://www.scielo.br/ s c i e 1 o.ph p s c ri pt=s ci arttext \& pid=S 1516 $35982009001200004 \& \operatorname{lng}=$ en $\&$ nrm=iso $>$. Acesso em: 12 set. 2010. doi: $10.1590 /$ S1516-35982009001200004

PILLAR, V.D. Sampling sufficiency in ecological surveys. Abstracta Botânica, v.22, p.37-48, 1998. Disponível e m: $<$ ht t p://ecoqua.ecologia.ufrgs.br/arquivos/ Reprints\&Manuscripts/Pillar_1998_AbtractaBot.pdf $>$. Acesso em: 12 set. 2010.

PILLAR, V.D. MULTIV, Multivariate exploratory analysis, randomization testing and bootstrap resampling. Porto
Alegre: Departamento de Ecologia, UFRGS, 2004. Disponível em: <http://ecoqua.ecologia.ufrgs.br/ 2004>. Acesso em: 12 set. 2010 .

POND, K.R. et al. Passage of chromium-mordanted and rare earth-labeled fiber: time of dosing kinetics. Journal Animal Science, v.67, n.4, p.1020-1028, 1989.

PRACHE, S.; ROGUET, C. Influence de la structure du couvert sur le comportement d'ingestion. ClermontFerrand: Institut National de la Recherche Agronomique, 1996. p.22-24. (Rapport d'Activité 1992-1995).

ROOK, A.J. Principles of foraging and grazing behaviour. In: HOPKINS, A. (Ed.). Grass, its production an utilization Hurley: British Grassland Society, 2000. p. 229-246.

SAS Institute. Statistical analysis system user's guide. Version 8.2. Cary, 2001. CDROM.

SEARLE, K.R. et al. It's the "foodscape", not the landscape: using foraging behavior to make functional assessments of landscape condition. Israel Journal of Ecolology \& Evolution. v.53, p.297-316, 2007.

SOLLENBERGER, L.E., BURNS, J.C. Canopy characteristics, ingestive behavior and herbage intake in cultivated tropical grasslands. In: INTERNATIONAL GRASSLAND CONGRESS, 19., 2001, São Pedro. Proceedings... Piracicaba: Fealq, 2001. p.321-327.

UNGAR, E.D. Ingestive behaviour. In: HODGSON, J.; ILLIUS, A.W. The ecology and management of grazing systems. Oxon: CABI, 1996. p.185-218. 